\title{
Sistema de seguridad ante sobrecargas en un subsolador
}

\author{
Phil A. Pontoja C. \\ Ruth E. Cano B.** \\ José l. García M.**
}

\author{
Recibido: 03/08/2015 - Aceptado: 11/12/2015 \\ DOI: 10.22395/rium.v15n28a11
}

\begin{abstract}
Resumen
Actualmente, la tecnificación del campo ha surgido como una respuesta a la necesidad de mejorar el rendimiento de la producción de los campos, principalmente en países agrícolas como Colombia, por aspectos como el incremento demográfico y la globalización económica, donde la labor de subsolado es la primera actividad realizada, la cual procura la descompactación del suelo para disminuir su resistencia y beneficiar el esparcimiento de las raíces de las plantas y el flujo de agua. Así, cualquier tipo de anormalidad en el desarrollo de esta actividad genera atrasos en las labores posteriores o disminuye el rendimiento del cultivo. Este artículo muestra los resultados de una investigación tecnológica, enfocados en la adecuación mecatrónica de un implemento de subsolado el cual se planteó bajo un procedimiento estructurado de diseño que garantiza las adaptaciones tecnológicas asegurando el funcionamiento de un sistema instrumentado de control con respuesta ante sobrecargas.
\end{abstract}

Palabras clave: dispositivo de seguridad, mecatrónica, sobrecarga, subsolador

Ing.; Escuela de ingeniería Mecánica, Universidad del Valle, Cali, Colombia. Calle 13 No 100-00 edificio 340 ofic.. 2011. Teléfono ofic.: (+2) 3212100 ext. 3234. Celular: 315204 2788. Correo: phil.pontoja@correounivalle. edu.co.

** Ing.; Escuela de ingeniería Mecánica, Universidad del Valle, Cali, Colombia. Calle 13 No 100-00 edificio 340 ofic. 2011. Celular: 300609 7178. Correo: ruth.cano@correounivalle.edu.co

*** PhD; Escuela de ingeniería Mecánica, Universidad del Valle, Cali, Colombia. Calle 13 No 100-00 edificio 340 ofic. 2011. Teléfono ofic.: (+2) 3212100 ext. 3234. Celular: 316744 2820. Correo: jose.i.garcia@correounivalle. edu.co. 


\title{
Security system for overloading of a subsoiler
}

\begin{abstract}
Currently, rural technological development has emerged as a response to the need for improving production efficiency in the fields, especially in agricultural countries such as Colombia due to aspects such as demographic growth and economic globalization; the use of a subsoiler is the first activity performed, intended to loosen soil as a way of reducing its resistance and allowing the spread of plant roots and flow of water. Hence, any type of abnormal circumstances in the development of this activity results in delays in future works or decrease of crop efficiency. This article shows the results of a technological research, focused on mechatronic adequacy of a subsoiler implement addressed under a structured design procedure that assures technological adaptations by assuring the operation of a control instrumented system with response to overloading.
\end{abstract}

Key words: Security device; mechatronics; overloading; subsoiler. 


\section{INTRODUCCIÓN}

La agricultura tradicional engloba técnicas agrarias utilizadas en distintas civilizaciones presentes de la memoria cultural. Este tipo de agricultura ocupa más del $70 \%$ de la población mundial activa [1]. Sin embargo, incentivado por el crecimiento constante de la población mundial, este tipo de agricultura ha evolucionado a una agricultura mecanizada. Así, a partir de 1892, con la implementación del tractor en las actividades agrícolas emergentes, técnicas mecanizadas fueron empleadas en la agricultura [2].

Así, varios autores han presentado sus trabajos con este objetivo [3 - 8]. Sin embargo, al nivel nacional, la tecnificación de las diferentes actividades agrícolas se puede considerar incipiente; esto fundamentado principalmente por los costos y la falta de experiencia en la adaptación de automatización en aplicaciones agrarias al nivel local. Por ejemplo, en la actividad de subsolado que descompacta el suelo permitiendo la permeabilidad de este y el crecimiento de las raíces de las plantas, se utilizan implementos que presentan estructuras rígidas, lo cual impide adecuarse a las variaciones de terreno, climatológicas, y ubicación geográfica, entre otros.

Este proyecto se centró en la mecanización del subsolado, mediante la integración de sensores de medición de fuerza que, en conjunto con un elemento de actuación, retiran el implemento del suelo ante una sobrecarga, considerando la restricción de energía hidráulica disponible por parte del tractor. De esta forma, el equipo de instrumentado concebido realiza el control de sobrecarga en el subsolador y registra las cargas de operación.

En la sección dos se describen los requisitos de diseño de la herramienta. La sección tres detalla el estado de carga del subsolador. La sección cuatro muestra los componentes mecánicos del sistema de medición de carga. De la sección cinco a la ocho se describen los modelos del sistema, su arquitectura, casos de uso, diagramas de actividades, estrategia de control y fuerzas de referencia, respectivamente. En la sección nueve se detalla el procedimiento de instrumentación que se llevó a cabo. Finalmente en la sección diez se muestran las conclusiones.

\section{REQUISITOS DE DISEÑO}

Considerando el estado de preparación de suelo necesario, en un ingenio azucarero de la región de Valle del Cauca, se requería una instrumentación del equipo de subsolado que permitiese la protección del implemento ante sobrecarga y el registro de cargas. Para el desarrollo de este proyecto se consideraron dos restricciones: i) el uso de un equipo de subsolado existente en el ingenio, y ii) el uso del potencial hidráulico ofrecido por el tractor que tira el equipo para levantar el subsolador. 
Considerando una variación imprevista en la rigidez del suelo, debida a un obstáculo, que ponga en riesgo la integridad física de los componentes del equipo de subsolado seleccionado en el ingenio (figura 1), por una sobrecarga, se planteó la necesidad de un sistema de respaldo al dispositivo mecánico de seguridad del equipo. Para esto, se evaluaron distintas soluciones de seguridad existentes teniendo en cuenta los siguientes aspectos: portabilidad del dispositivo, mantenimiento, confiabilidad, flexible ante adecuaciones. De esta forma, se debe garantizar la continuidad de operación de subsolado independientemente de la existencia de un obstáculo.

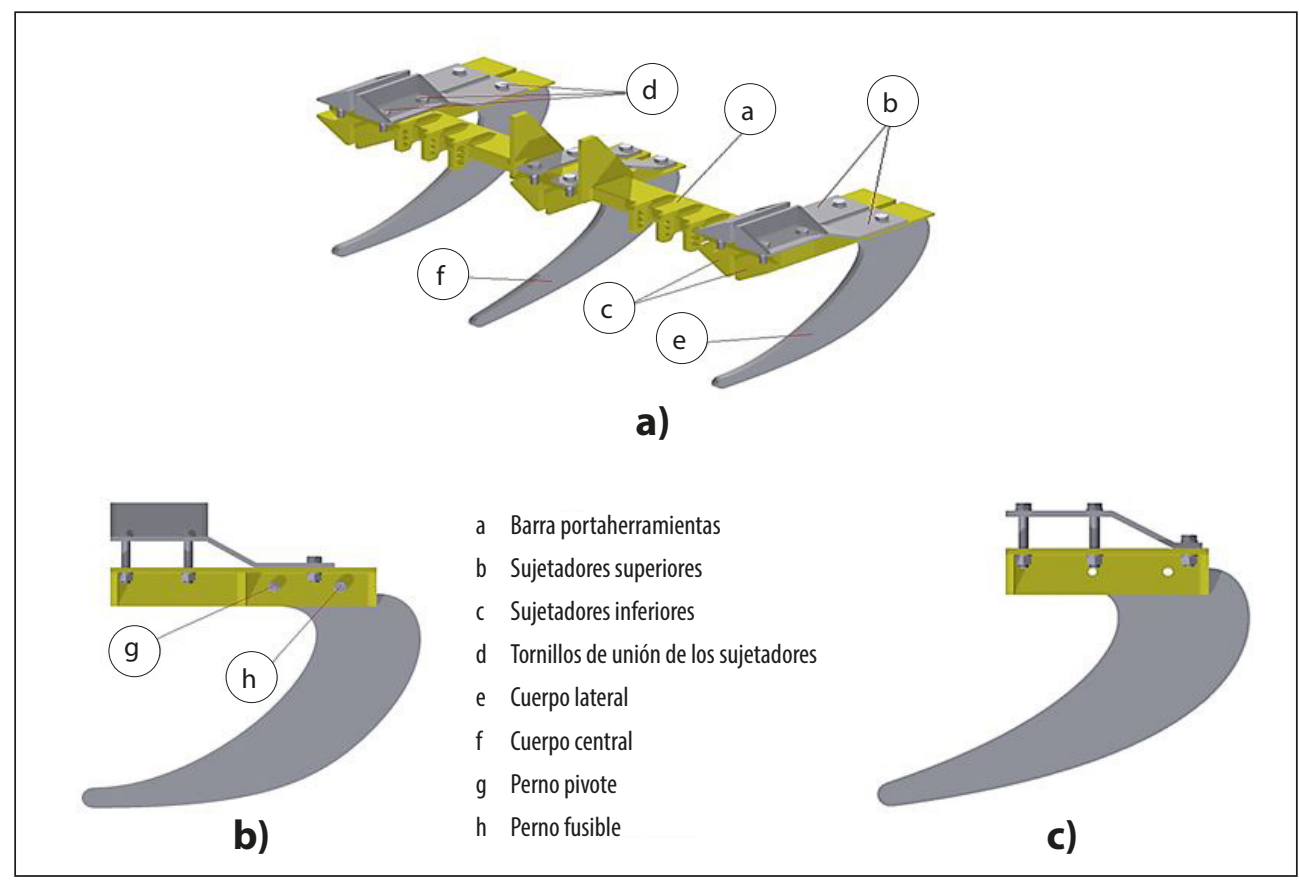

Figura 1. Conexión de los cuerpos al marco: a) tres cuerpos conectados al marco. b) uno de los cuerpos laterales con sujetadores que lo acoplan la barra. c) cuerpo central con sujetadores.

Fuente: elaboración propia

Basado en los aspectos anteriores y en la información de los dispositivos de seguridad encontrados en la literatura, una matriz de decisión permitió seleccionar la alternativa adecuada para el sistema de respaldo ante una falla del sistema instrumentado. La tabla 1 muestra una valoración de cada una de las alternativas de sistemas de seguridad con relación a los aspectos básicos que estos deben cumplir. La columna dos muestra la calificación de cada aspecto (en un intervalo de 0 a 10) según su nivel de importancia. La columna tres muestra el peso porcentual de cada aspecto, el cual se obtuvo dividiendo la calificación individual de cada una de ellas por la suma de las calificaciones. La valoración de cada alternativa de seguridad con respecto a 
cada aspecto se hizo en una escala de 1 a 10, siendo 10 un indicador de excelencia de desempeño en un aspecto específico. La valoración global de cada alternativa se obtuvo multiplicando cada calificación de estas respecto al peso porcentual del aspecto relacionado y sumándolos al final. La valoración mostró que la mejor alternativa fue el perno fusible sometido a cortante.

Tabla 1. Matriz de decisión del sistema de respaldo

\begin{tabular}{|l|c|c|c|c|c|c|c|}
\hline & & $\begin{array}{c}\text { Peso porcentual } \\
\text { del aspecto }\end{array}$ & Ballesta & Muelle & $\begin{array}{c}\text { Cilindro } \\
\text { hidroneumático }\end{array}$ & $\begin{array}{c}\text { Fusible a } \\
\text { cortante }\end{array}$ & $\begin{array}{c}\text { Fusible a } \\
\text { tensión }\end{array}$ \\
\hline Portabilidad & 7 & 18.92 & 6 & 7 & 7 & 10 & 10 \\
\hline Mantenimiento & 9 & 24.32 & 7 & 6 & 5 & 10 & 9 \\
\hline Confiabilidad & 8 & 21.62 & 9 & 8 & 10 & 6 & 6 \\
\hline $\begin{array}{l}\text { Flexibilidad de } \\
\text { adecuación }\end{array}$ & 6 & 16.22 & 6 & 8 & 8 & 10 & 10 \\
\hline Continuidad de trabajo & 7 & 18.92 & 9 & 9 & 10 & 5 & 5 \\
\hline Total & 36 & 100.00 & 7.46 & 7.51 & 7.9 & 8.19 & 7.95 \\
\hline
\end{tabular}

Fuente: elaboración propia

Un sumario de los requisitos del sistema se presenta a continuación:

1. El respaldo debe ser del tipo perno fusible sometido a cortante.

2. El registro de las fuerzas debe almacenarse en un dispositivo SD.

3. Se debe accionar el sistema de levantamiento hidráulico ante una sobrecarga.

4. Se debe avisar al operador por medio de una alarma visual y sonora ante el evento de una sobrecarga.

\section{ESTADO DE CARGA DE LOS CUERPOS DEL SUBSOLADOR}

Para el planteamiento de las modificaciones geométricas en la estructura del subsolador que permitiese una adaptación de los pernos fusibles y celdas de carga, fue necesario identificar el estado de cargas del cuerpo del subsolador que causarán una falla en el fusible. A través de estas fuerzas, se identificó el esfuerzo máximo soportado por el cuerpo en su configuración inicial. Dicho esfuerzo sirvió de referencia máxima para los cuerpos de los subsoladores en la configuración conceptual propuesta. Para esto, se consideró realizar dos tipos de modelos estáticos estructurales utilizando el método de elementos finitos (FEM). 
En el primer modelo se varió el ángulo de fricción de la herramienta con un suelo seco, y se incrementó la fuerza total P, basada en el modelo teórico de Reece, de tal manera que la fuerza de reacción debido al perno fusible se igualara con la necesaria para hacerlo fallar [9, 10]. En el segundo modelo es considerada una fuerza de operación basada en el modelo matemático de la fuerza que actúa en la herramienta debido al suelo con datos de zonas del Valle $[11,12]$. Esto, para verificar los resultados del primer modelo. A continuación, se muestran los resultados para el cuerpo lateral.

En los modelos FEM se empleó un enmallado con elementos hexaédricos de 20 nodos y cuñas de 15 nodos, donde cada nodo posee 3 grados de libertad de desplazamiento que corresponden a las direcciones X, Y y Z. Adicionalmente, presentan refinamientos de la malla en las caras de los orificios. Las condiciones de borde fueron una fuerza aplicada sobre una línea en la parte inferior del cuerpo y dos soportes que solo permitan compresión en cada agujero del cuerpo, emulando la interacción del suelo-herramienta y la de los pernos, respectivamente.

\subsection{Esfuerzos máximos en los cuerpos del subsolador variando el ángulo de fricción y la magnitud de la fuerza total $P$}

Como se puede apreciar en la figura 2, el esfuerzo equivalente de Von Mises máximo del cuerpo, $447.42 \mathrm{MPa}$, se encuentra en el agujero donde va el perno pivote (figura 2b), y casi duplicó al esfuerzo de fluencia del material del cuerpo, el cual tiene un valor de $250 \mathrm{MPa}$. Por este motivo, se concluyó que el perno fusible 1 1/8-7 UNC-2A de clase SAE 5 utilizado en el subsolador seleccionado no era adecuado para la seguridad del cuerpo lateral. Así, se tomó la decisión en este proyecto de realizar todos los modelos con un perno fusible de menor resistencia como lo es el perno 1 1/8-7 UNC-2A de clase SAE 2.

Las simulaciones se realizaron para distintos ángulos; los resultados se presentan en la Tabla 2, donde se muestra el ángulo de fricción, $\delta$, la fuerza total, $P$, debido a la interacción suelo-herramienta, la reacción en el cuerpo de la simulación, $F_{f}$, debido al perno fusible y la que generaría falla en el perno, $2 F_{R}$, el esfuerzo equivalente de Von Mises máximo, $\sigma_{V}$, y el factor de seguridad ante fluencia, $F S$, del cuerpo. De esta tabla se puede apreciar que el mayor esfuerzo efectivo se presenta para un ángulo de fricción de $11^{\circ}$ y una fuerza $\mathrm{P}$ de $25621 \mathrm{~N}$, el cual tiene un valor de $215.32 \mathrm{MPa}$. 

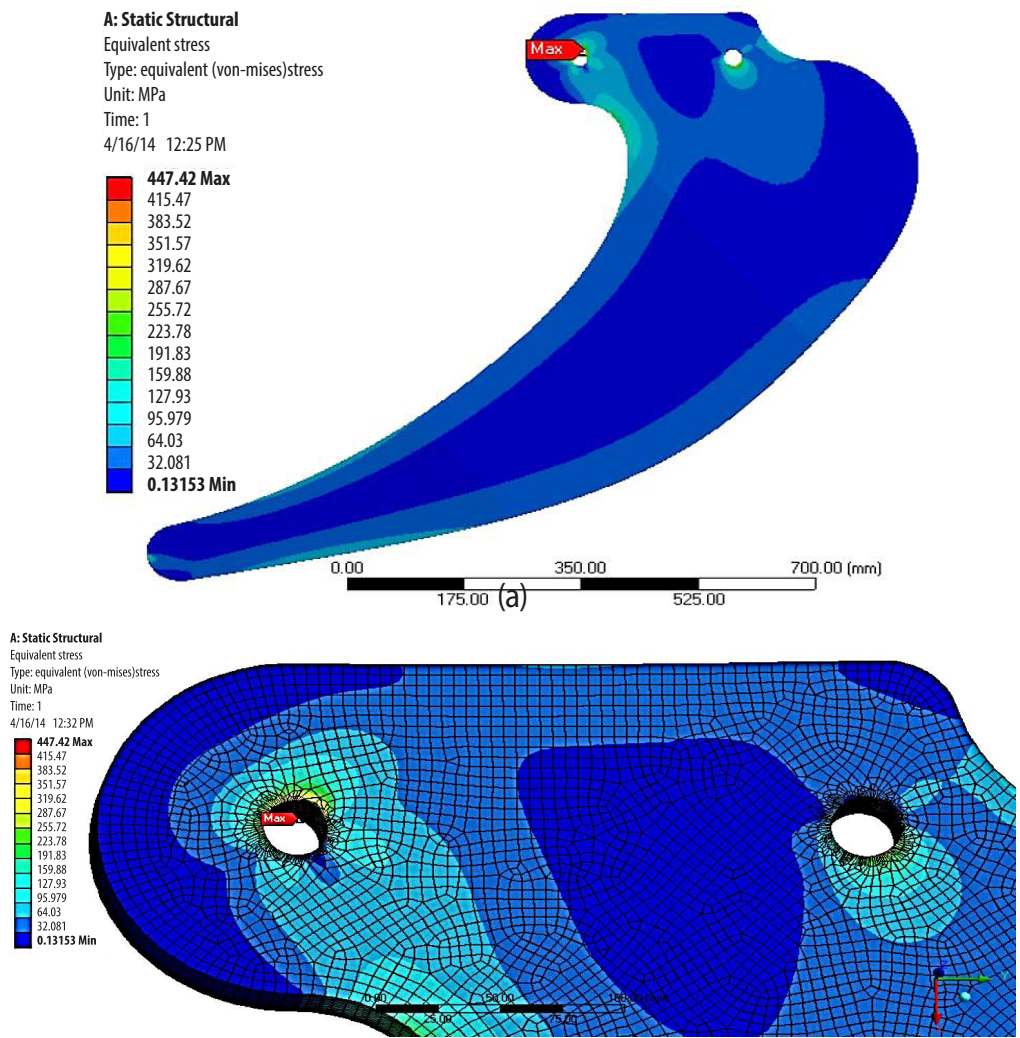

(b)

Figura 2. Esfuerzo equivalente de Von Mises para un ángulo de fricción de $22.01276132^{\circ}$ y el perno fusible de grado SAE 5, en a) Distribución del esfuerzo equivalente de Von Mises en el cuerpo y en b) Esfuerzo equivalente máximo en el cuerpo.

Fuente: elaboración propia

Tabla 2. Resultados del modelo para el cuerpo lateral a distintos ángulos de fricción

\begin{tabular}{|c|c|c|c|c|c|c|c|}
\hline \multirow{2}{*}{$\delta\left(^{\circ}\right)$} & Fuerza & \multicolumn{3}{|c|}{$F f(N)$} & \multirow{2}{*}{ 2FR $(N)$} & $\sigma_{\text {vmáx }}(M P a)$ & $F S$ \\
\cline { 3 - 5 } & $P(N)$ & $\begin{array}{c}\text { Componente } \\
\text { Horizontal }\end{array}$ & $\begin{array}{c}\text { Componente } \\
\text { Vertical }\end{array}$ & Total & & \\
\hline 11.00 & 25621 & 23992.90 & 114926.80 & 117404.56 & & 215.32 & 1.16 \\
\hline 14.00 & 25369 & 23138.54 & 115103.35 & 117406.02 & & 204.04 & 1.23 \\
\hline 17.00 & 25192 & 22312.19 & 115267.55 & 117407.17 & \multirow{2}{*}{117407.86} & 201.51 & 1.24 \\
\hline 22.00 & 25052 & 20972.67 & 115518.68 & 117407.07 & & 199.46 & 1.25 \\
\hline 22.01 & 25052 & 20969.44 & 115519.81 & 117407.59 & & 199.46 & 1.25 \\
\hline
\end{tabular}

Fuente: elaboración propia 


\subsection{Esfuerzos máximos en los cuerpos del subsolador basados en las propiedades físicas del suelo de la región de trabajo}

Para identificar el esfuerzo máximo en los cuerpos del subsolador en la región de operación, se calcularon las fuerzas en dirección vertical y horizontal que actúan en la herramienta con ayuda del modelo planteado por Reece, considerando condiciones extremas de suelo seco y de densidad crítica de texturas de suelo, siendo estas $10.04 \mathrm{kN}$ y $11.96 \mathrm{kN}$, respectivamente. Para el modelo FEM se usó enmallado similar e iguales condiciones de borde que las descritas en la sección anterior.

Los esfuerzos fueron de 122.09 MPa para el cuerpo lateral, para una carga en una operación crítica. Como criterio de diseño para el sistema de seguridad de los cuerpos se tomaron los valores de esfuerzo del primer modelo, ya que representa el estado de sobrecarga.

\section{COMPONENTES MECÁNICOS DEL SISTEMA DE MEDICIÓN DE CARGA}

La disposición actual de los cuerpos del subsolador se muestra en la figura 3. Aprovechando esta configuración y realizado el análisis estructural se aseguró que el componente mecánico vulnerable sea el fusible, y se consideró la fuerza cortante que hace fallar al fusible como parámetro para activar el mecanismo de seguridad ante sobrecarga.

El uso de celdas de carga permitió cuantificar la carga cortante en el fusible y, considerando el estado isostático del cuerpo del subsolador, se determinaron indirectamente las cargas horizontal y vertical debido a la interacción suelo-herramienta. Adicionalmente, se concibieron dos elementos que conectan un cuerpo subsolador a los sujetadores superiores, los cuales se encuentran sometidos a un estado uniaxial de carga debido a las dos cargas aplicadas y a la libertad de giro en sus extremos, donde, la carga axial interna de uno de estos componentes es igual a la fuerza cortante interna en el perno fusible (ver figura 3).

Los medidores de deformación están organizados como componentes de un puente de Wheatstone, localizados en los elementos mecánicos de unión de los sujetadores y los cuerpos, respectivamente, donde se mide la carga axial en estos elementos. La figura 3a muestra al subsolador con algunas modificaciones geométricas en su estructura; se recortaron los sujetadores superiores e inferiores para poder poner un elemento mecánico que dirige la fuerza en el fusible. También, se agregaron placas con forma de orejas con sus respectivos refuerzos en los sujetadores superiores de los cuerpos laterales de manera que se pudiera enlazar el elemento que dirige la fuerza con el sujetador. En las figuras $3 \mathrm{~b}$ y $3 \mathrm{c}$ se muestran las vistas laterales de los cuerpos lateral y central, respectivamente. 


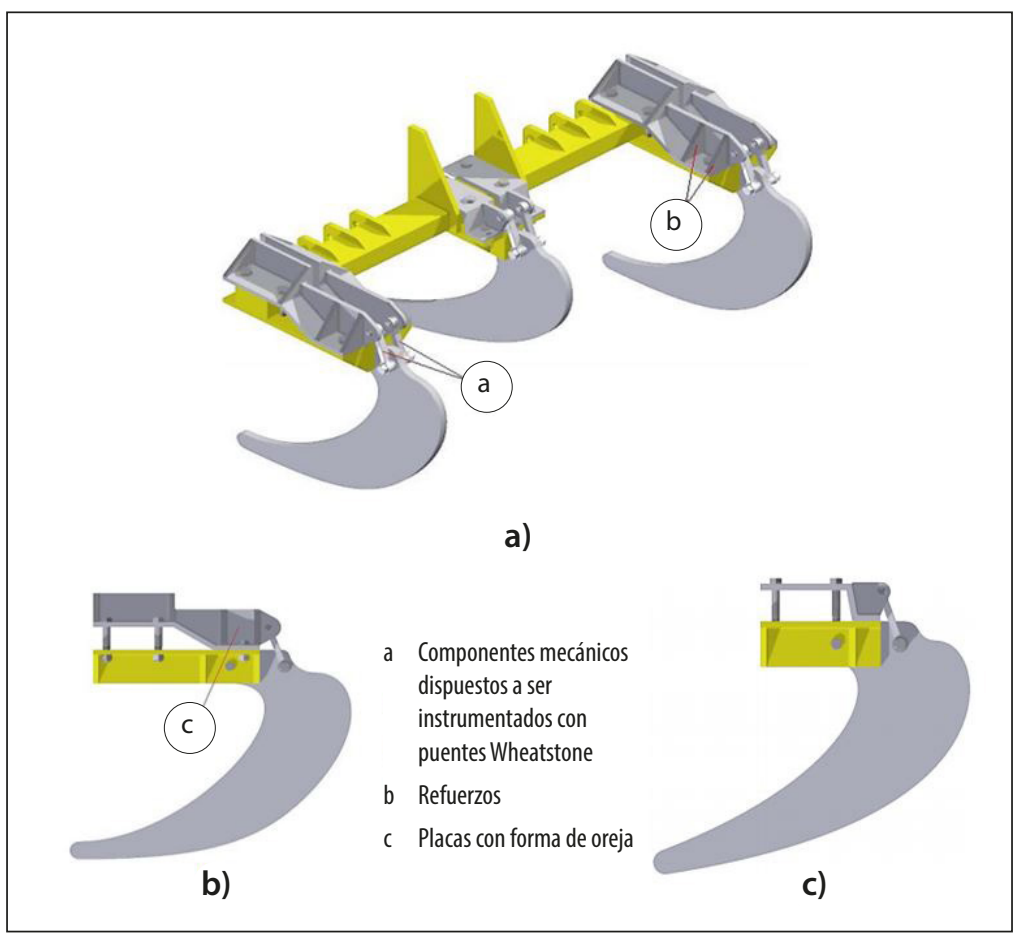

Figura 3. Diseño conceptual de los componentes mecánicos de medición de carga.

a) Subsolador con cambios geométricos en sus sujetadores. b) Vista lateral del cuerpo lateral.

c) Vista lateral del cuerpo central.

Fuente: elaboración propia

\section{ARQUITECTURA DEL SISTEMA INSTRUMENTADO}

La manera en que las partes que constituyen un sistema se organizan e interactúan entre sí se conoce como arquitectura, y una forma de representarla es mediante un diagrama IDEF0. Su lenguaje gráfico se presenta de una manera organizada y sistemática para facilitar la compresión y el análisis [13]. Adicionalmente, permite especificar requisitos, apoyar el diseño de subniveles del sistema y de actividades de integración [13]. Así, se representó una arquitectura del sistema mediante módulos funcionales. En la figura 4 se observa la representación global del sistema instrumentado para el control de sobrecarga (SICS), donde las entradas necesarias para que el sistema actúe, lado izquierdo, en este caso, son suministros de distintos tipos de energía. Del lado derecho se encuentran las salidas del sistema, las cuales son una alarma sonora y otra visual, el movimiento del implemento de arado, una retroalimentación visual, y un registro de las fuerzas que actúan en el implemento debido a su interacción con el suelo. En la parte superior se encuentran las condiciones de control que configuran al sistema para producir unas determinadas salidas deseadas. En este caso, estas condiciones son un 
"encendido 1 y 2", un "inicio 1 y 2", un reset, datos de suelo y la presión hidráulica de referencia.

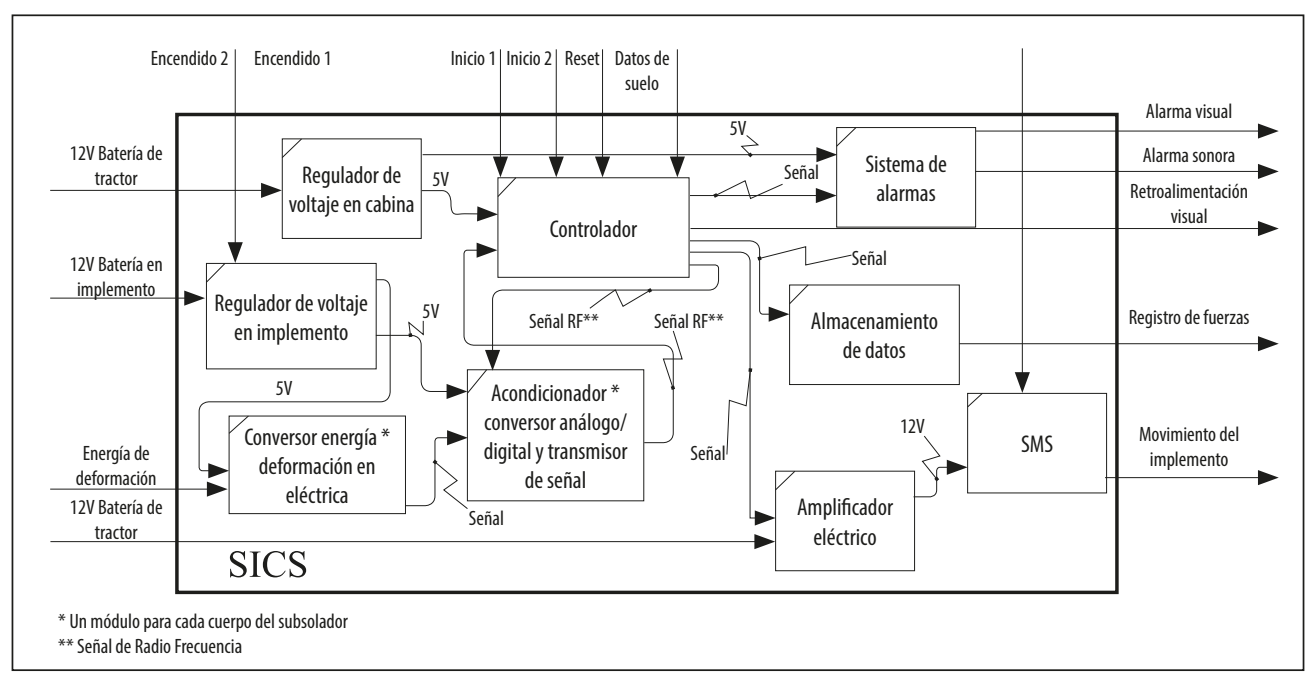

Figura 4. Representación de las funciones u operaciones internas del SICS.

Fuente: elaboración propia.

\section{CASOS DE USO DEL SISTEMA INSTRUMENTADO}

El diagrama de casos de usos de UML es uno de los modelos frecuentemente utilizados y se encuentra clasificado dentro de los diagramas de comportamiento [14]. Este modelo es una exhibición de la interacción entre uno o varios actores con el sistema, donde cada caso de uso representa un determinado requerimiento funcional [14]. Así, el actor que interactúa con el sistema instrumentado es definido como operario (ver figura 5). Se consideraron los casos de uso: adquirir datos, comparar datos, almacenar datos y proteger implemento de arado, encerrados en elipses. Las relaciones entre sí y con actor son representadas con flechas. Los tipos de relaciones entre los casos de uso de este sistema fueron include y extend. La primera tiene como objetivo que un caso de uso llame a otro para su funcionamiento normal o, en otras palabras, lo incluye en su funcionalidad. En la segunda, se considera un escenario específico para que un caso de uso amplíe o extienda la funcionalidad de otro caso de uso. En la tabla 3, se muestra la descripción del escenario correspondiente al diagrama de casos de uso. 


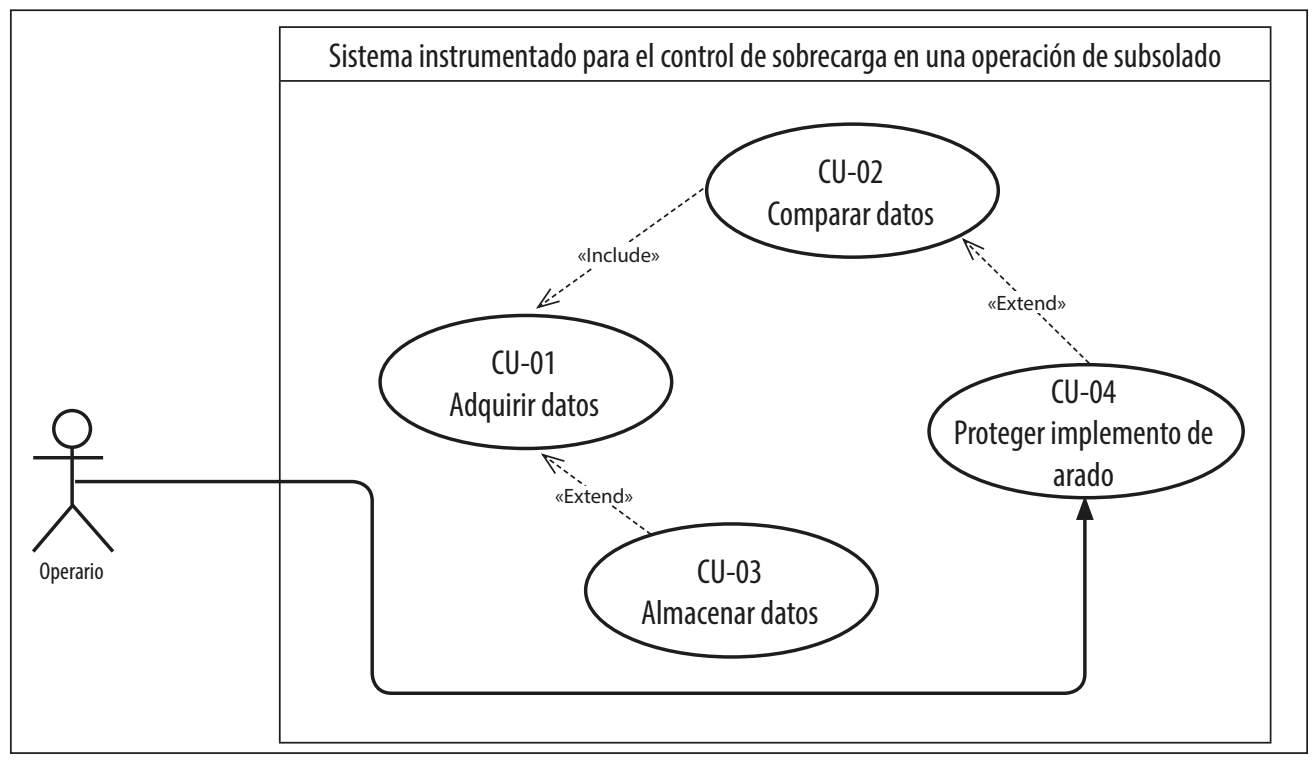

Figura 5. Diagrama de casos de uso del sistema.

Fuente: elaboración propia

Tabla 3. Caso de uso adquirir datos

Nombre: Adquirir datos (CU-01)

Autor: Phil Anderson Pontoja

Fecha: 26/06/2014

Descripción:

El operario presiona el botón inicio 1 y el sistema comienza a recibir datos de deformación para asociarlos a fuerza, y si opcionalmente el operario presiona el botón inicio 2, el sistema registrara los datos de suelo suministrados por el operario.

Actores: Operario.

Precondiciones:

El subsolador se encuentra incorporado en el suelo y el sistema debe estar energizado. Flujo normal: 
1. El operario pulsa el botón inicio 1.

2. El sistema recibe una señal del transductor y las va asociando con las fuerzas que actúan en el elemento mecánico del mismo.

Flujo alternativo:

1A. El operario pulsa el botón inicio 1 y 2.

3. El sistema solicita en pantalla el coeficiente de adhesión del suelo-herramienta y el ángulo de fricción suelo-herramienta.

4. El operario digita el coeficiente de adhesión del suelo-herramienta y el ángulo de fricción suelo-herramienta.

6. El sistema muestra en pantalla los datos digitados.

7. El operario verifica los datos, realiza correcciones si es necesario y los introduce.

8. El sistema almacena los datos introducidos por el operario.

9. El sistema se mueve hacia el caso de uso CU-03.

Poscondiciones:

Fuente: elaboración propia

\section{DIAGRAMAS DE ACTIVIDADES}

Una vez establecida la arquitectura sistema y su interacción con el operario a través de los casos de uso, se describió el flujo de control por medio de diagramas de actividades de UML [15].

A manera de ejemplo, en la figura 6 se muestra el diagrama de actividad "adquirir datos", donde el flujo se da para cada cuerpo del subsolador. 


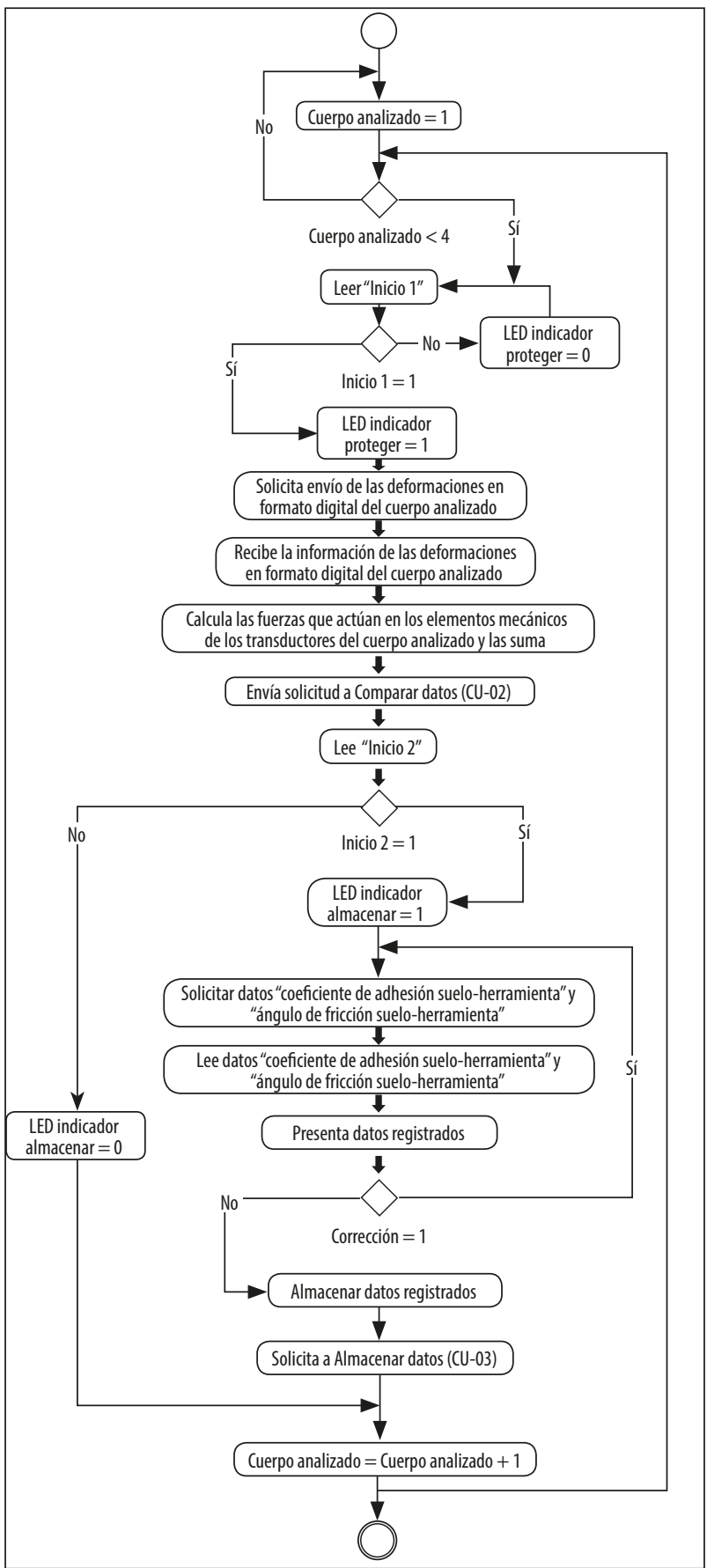

Figura 6. Diagrama de actividad adquirir datos.

Fuente: elaboración propia 
El operario debe presionar el botón "inicio 1" para que la actividad se lleve a cabo; opcionalmente, el operario puede presionar el botón "inicio 2" para una secuencia adicional de acciones en la actividad. La secuencia comienza con la lectura acerca del estado del botón "inicio 1" identificado con una variable con el mismo nombre. Si esta es distinta de 1, se desenergizará el LED indicador proteger, y se continuará leyendo de forma cíclica el valor de "inicio 1". Caso contrario, si "inicio 1" es igual a 1 se energizará el LED indicador proteger, se solicitará la información de las deformaciones, se recibirá la información, se calculará la fuerza que siente el cuerpo analizado debido a los elementos mecánicos de los transductores y se enviará el dato de la fuerza a la actividad "comparar datos". Una vez las acciones de comparar datos terminen, la actividad "adquirir datos" hará lectura de la variable "inicio 2". Si "inicio 2" es distinta de 1 se desenergizará el LED indicador almacenar, y la actividad se realizará para el siguiente cuerpo del subsolador. Por el contrario, si "inicio 2" es igual a 1 se energizará el LED indicador almacenar, se solicitarán datos de suelo al operario, se leerán los datos, se presentarán los datos digitados y se dará una opción de corrección de los mismos. Si la variable "corrección" es igual a 1, se volverán a ejecutar todas las acciones desde solicitar datos de suelo al operario. Caso contrario, se almacenarán los datos de suelo y se enviará una solicitud a la actividad "almacenar datos" para que realice sus acciones. Por último, una vez "almacenar datos" finalice, la actividad "adquirir datos" realizará las acciones para el siguiente cuerpo del subsolador.

\section{ESTRATEGIA DE CONTROL}

La estrategia de control implementada en este proyecto se puede clasificar dentro de la categoría de sistemas instrumentados de seguridad (SIS). Según [16], estos ejecutan una acción automática para mantener la planta en un estado seguro, o para llevarla a un estado seguro, cuando se presentan condiciones anormales. En este contexto, las acciones derivadas por este este tipo de control presentan un nivel de prioridad superior a las del nivel de control operacional encargadas de las condiciones normales de operación. La figura 7 presenta un esquema general de control planteado en este proyecto. Este está integrado por dos bucles, uno de posición y otro de SIS. El bucle de posición es uno operacional y tiene como objetivo asegurar un nivel de penetración del implemento subsolador, cuantificado por un sensor de posición LVDT, o de levantarlo cuando se encuentre en la operación de transporte. Para esto, se dispone de una válvula proporcional, integrada por un controlador PID y una válvula hidráulica, la cual ejerce una acción de control sobre el cilindro hidráulico basada en la señal de referencia voltaje proveniente de un joystick. 


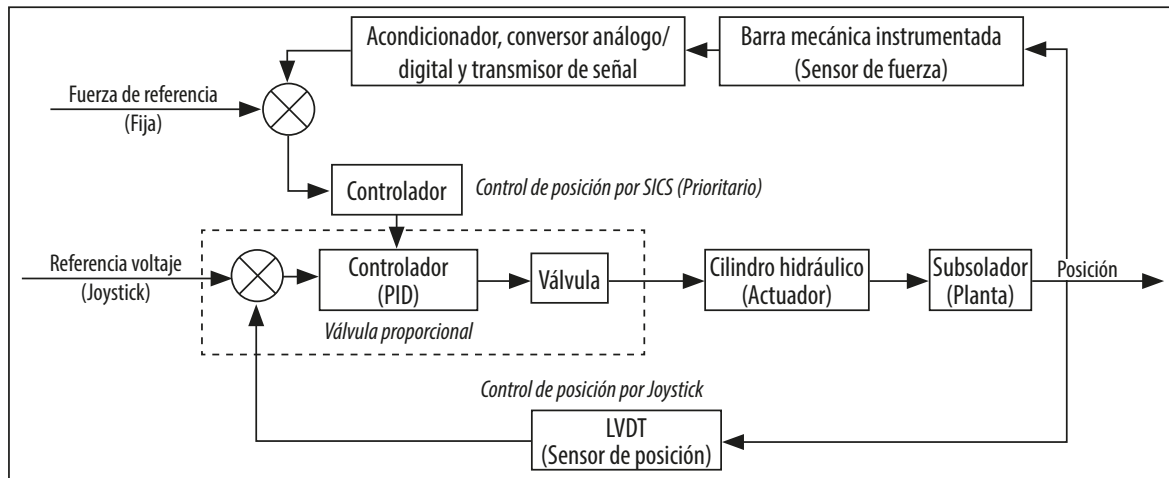

Figura 7. Bucles de control del SICS y del mando de posición por joystick.

Fuente: elaboración propia

El bucle SIS tiene como propósito asegurar una posición de levantamiento del subsolador y enviar una señal de alerta en caso de que el fusible mecánico del subsolador alcance un nivel crítico de esfuerzo. En este caso, el bucle SIS acciona la válvula proporcional. Como consecuencia, la válvula proporcional inhibe la acción de control del bucle operacional y levanta de forma urgente el subsolador.

En la figura 8 se presenta la válvula proporcional que garantiza el desplazamiento del cilindro hidráulico, por medio de un dispositivo electrónico que implementa un controlador PID, el cual es realimentado por medio de una señal proveniente de un LVDT.

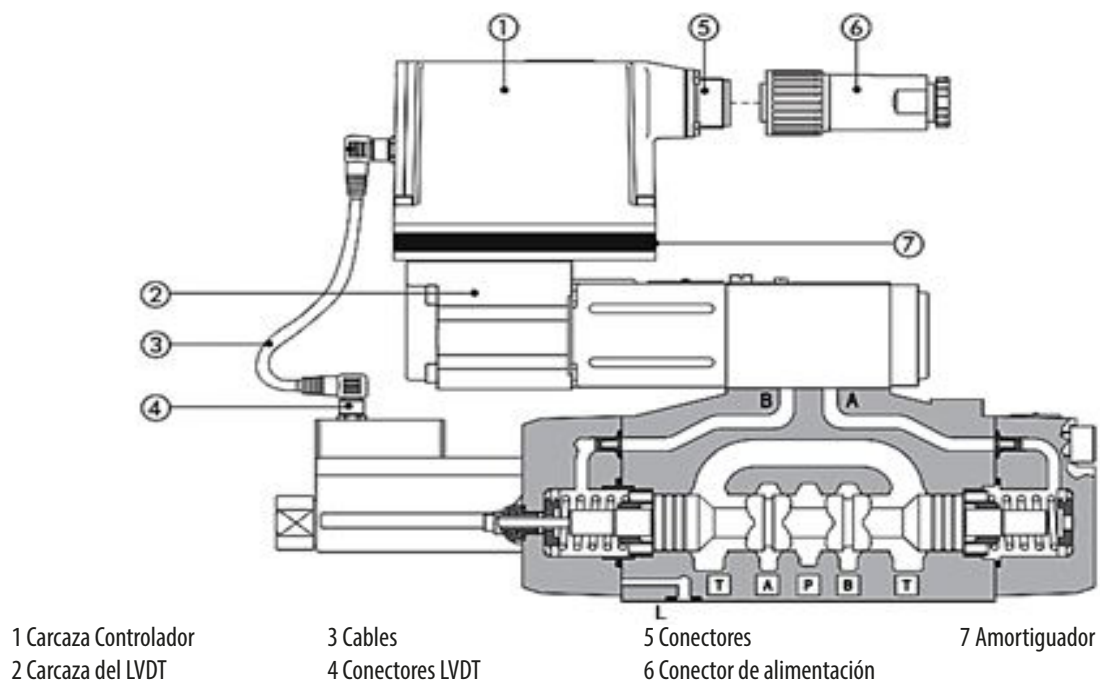

Figura 8. Válvula proporcional con sensor de posición LVDT.

Fuente: [17] 
La barra instrumentada está integrada por un componente mecánico calibrado, diseñado en la sección tres y un puente de wheatstone formado con medidores de deformación SG-2/350-LY47 de la empresa Omega. Este constituye el módulo de conversión energía de deformación en eléctrica del SICS. El módulo de acondicionador, conversión análoga/digital y transmisor de señal es presentado en la Figura 9, y está integrado fundamentalmente por un conversor análogo digital ADS1232, un microcontrolador PIC18F2X2, y un medio de transmisión inalámbrica XBEE-PRO, respectivamente.

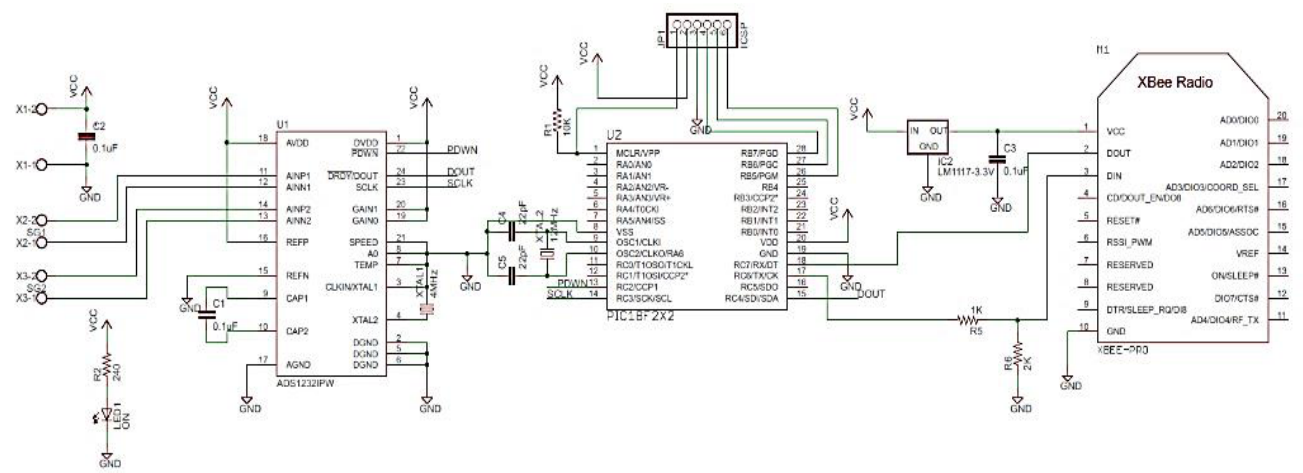

Figura 9. Módulo de acondicionamiento, conversión y transmisión.

Fuente: elaboración propia

El modulo controlador es presentado en la Figura e integrado por un componente de recepción inalámbrica XBEE-PRO, un microcontrolador PIC18F4X2 y un socket de memoria SD.

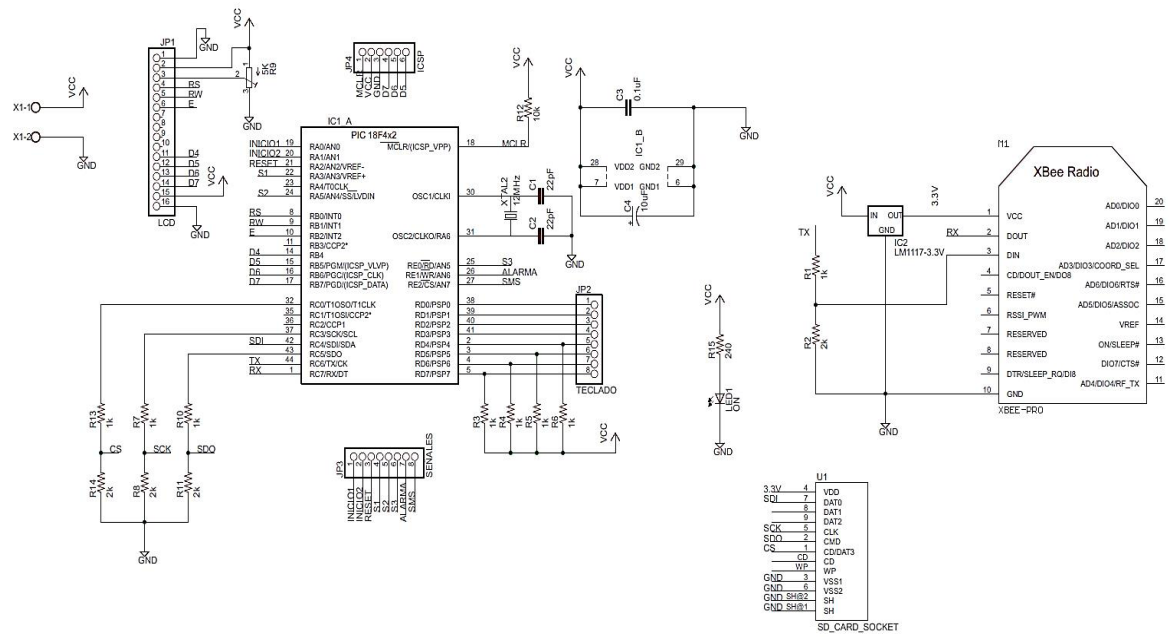

Figura 10. Módulo controlador.

Fuente: elaboración propia 


\section{FUERZA DE REFERENCIA PARA ACTIVAR EL SICS}

La fuerza máxima que soporta el fusible mecánico de los cuerpos subsoladores antes de su falla es de $58703.93 \mathrm{~N}$. Adicionalmente, el valor máximo de operación para el fusible del cuerpo lateral, teniendo en cuenta las condiciones de una operación crítica en los suelos de la región, es de 36606.6 N. Considerando los valores anteriores se definió que la fuerza de referencia en el SICS corresponde al $80 \%$ de la fuerza máxima que soporta el fusible mecánico antes de su falla, y garantiza la acción de subsolado en una operación crítica en los suelos de la región sin obstáculos.

\section{REGISTRO DE LA CARGA HORIZONTAL Y VERTICAL DEBIDO A LA INTERACCIÓN SUELO-HERRAMIENTA}

Considerando la barra instrumentada en un estado de esfuerzos axial debido a una sobrecarga, su parte intermedia registra una deformación de $0.035016 \%$, figura 11 y figura 12. En esta situación se realizó una conexión de cuatro medidores de deformación a tracción como el presentado en la figura 13.

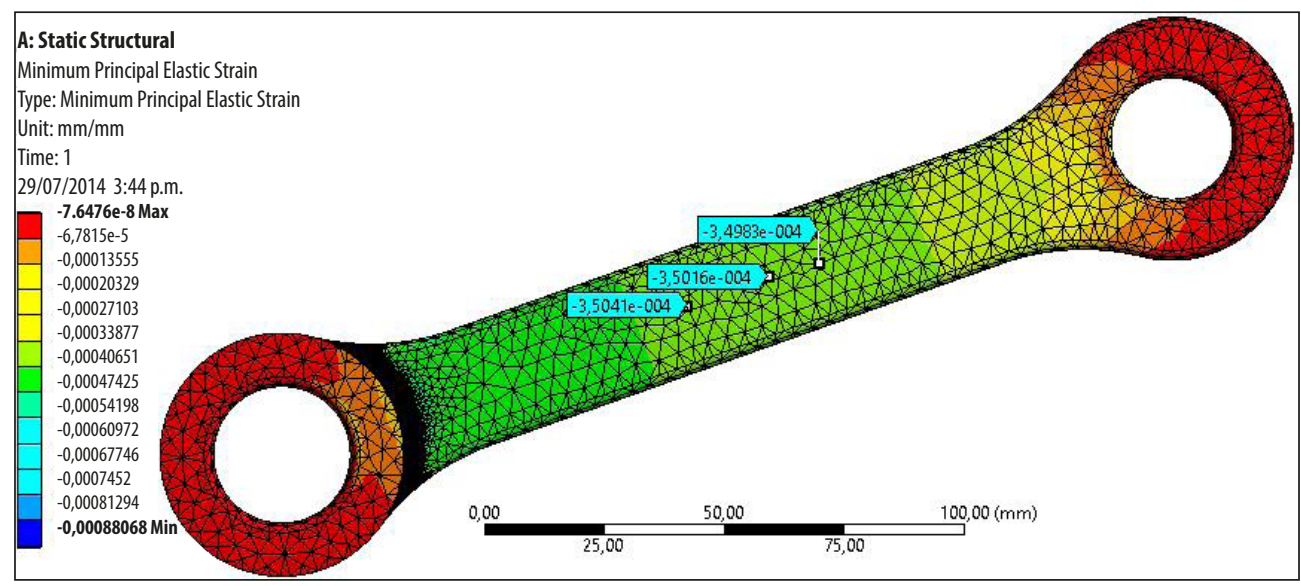

Figura 11. Deformaciones de la barra conectora en dirección de su eje.

Fuente: elaboración propia 


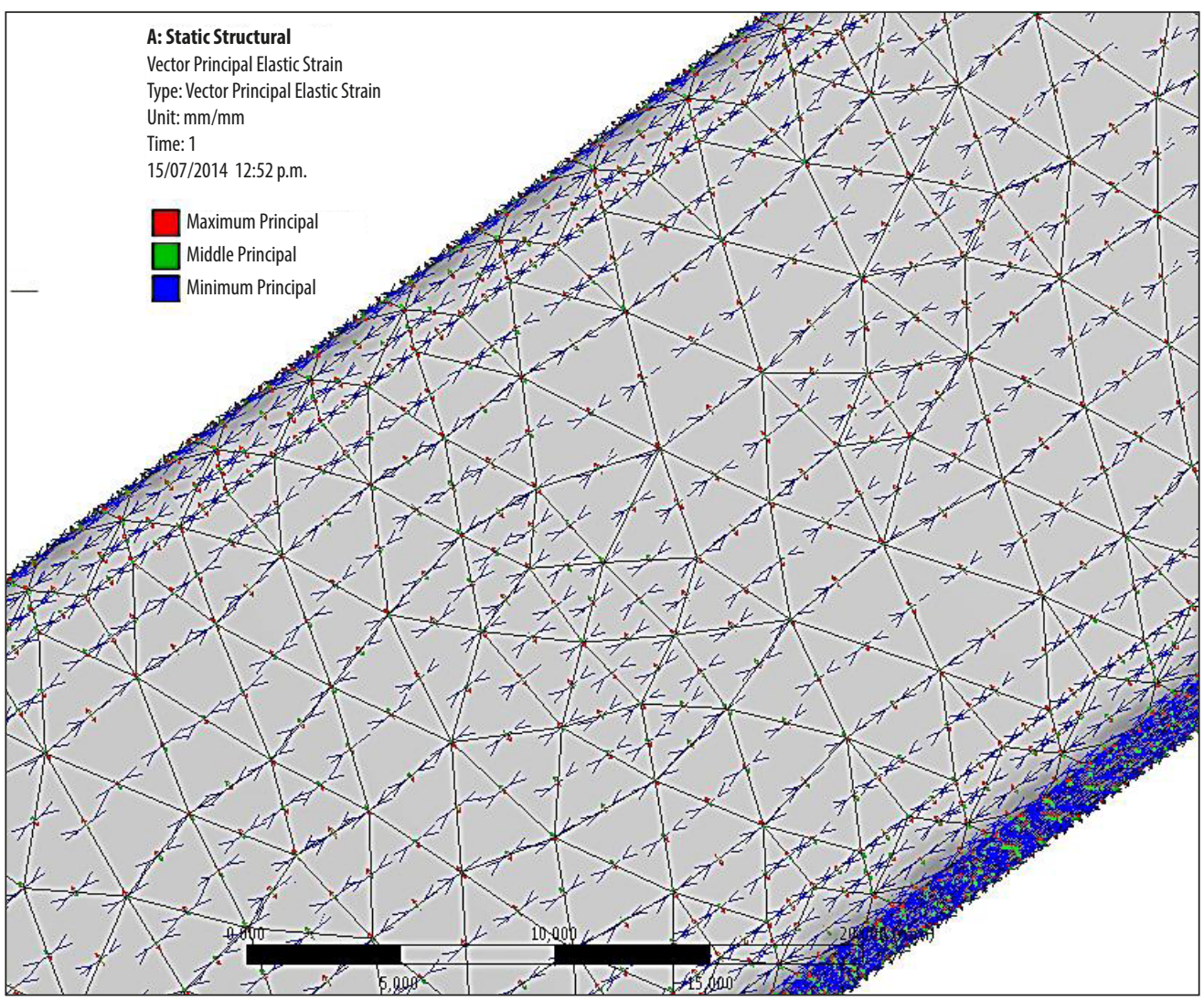

Figura 12. Dirección de las deformaciones mínimas principales de la barra.

Fuente: elaboración propia

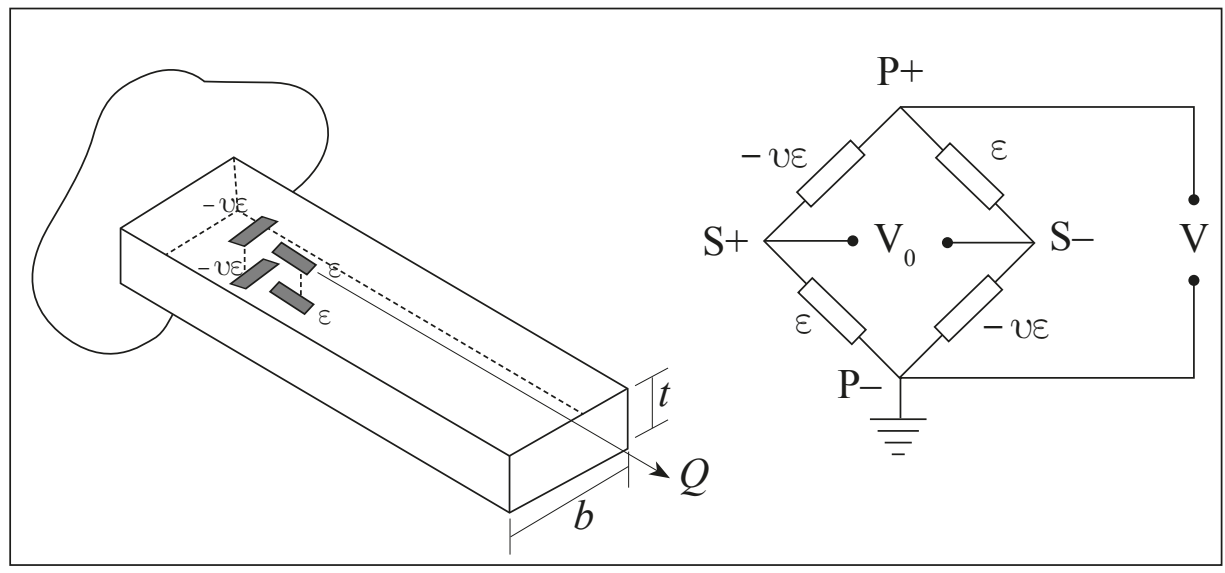

Figura 13. Conexiones del puente de Wheatstone.

Fuente: [18] 
La relación de salida del puente, $V_{0}$, y excitación del mismo, $V$, está definido por la ecuación (1):

$$
\frac{V_{0}}{V}=\frac{F \varepsilon(1+v)}{2}
$$

Donde: F, $\varepsilon$ y $V$ son el factor de galga, la microdeformación medida y el coeficiente de Poisson del material. Despejando se puede determinar las microdeformaciones como se ilustra en la ecuación (2):

$$
\varepsilon=\frac{2 V_{0}}{V F(1+v)}
$$

Y, el esfuerzo se relaciona con la deformación y la fuerza como se expresa en (3):

$$
\sigma=E\left(\varepsilon^{*} 10^{-6}\right)=\frac{Q}{A}
$$

Donde: $E$, $A$ y $Q$ son el módulo de Young del material, el área de sección transversal de la barra y la carga que actúa en dicha sección, respectivamente. Despejando $Q$ de la ecuación (3), se obtiene la fuerza en función de la microdeformación medida, ecuación (4).

$$
Q=A E\left(\varepsilon^{*} 10^{-6}\right)
$$

Para determinar la fuerza cortante que actúa a lo largo del perno fusible, se estima el promedio de las fuerzas en las dos barras instrumentadas que sujetan el tornillo de acuerdo con la ecuación (5).

$$
\frac{F_{f}}{2}=\frac{Q_{1}+Q_{2}}{2}
$$

Los subíndices 1 y 2 para $Q$ indican las fuerzas transmitidas por cada una de las barras,

la cual es calculada por el módulo controlador a fin de compararla con la fuerza de referencia para así proteger el implemento de arado ante una sobrecarga.

El medidor de deformación seleccionado fue SG-2/350-LY47 de la empresa Omega con un factor de galga $F$ de 2.0, figura 14 . 


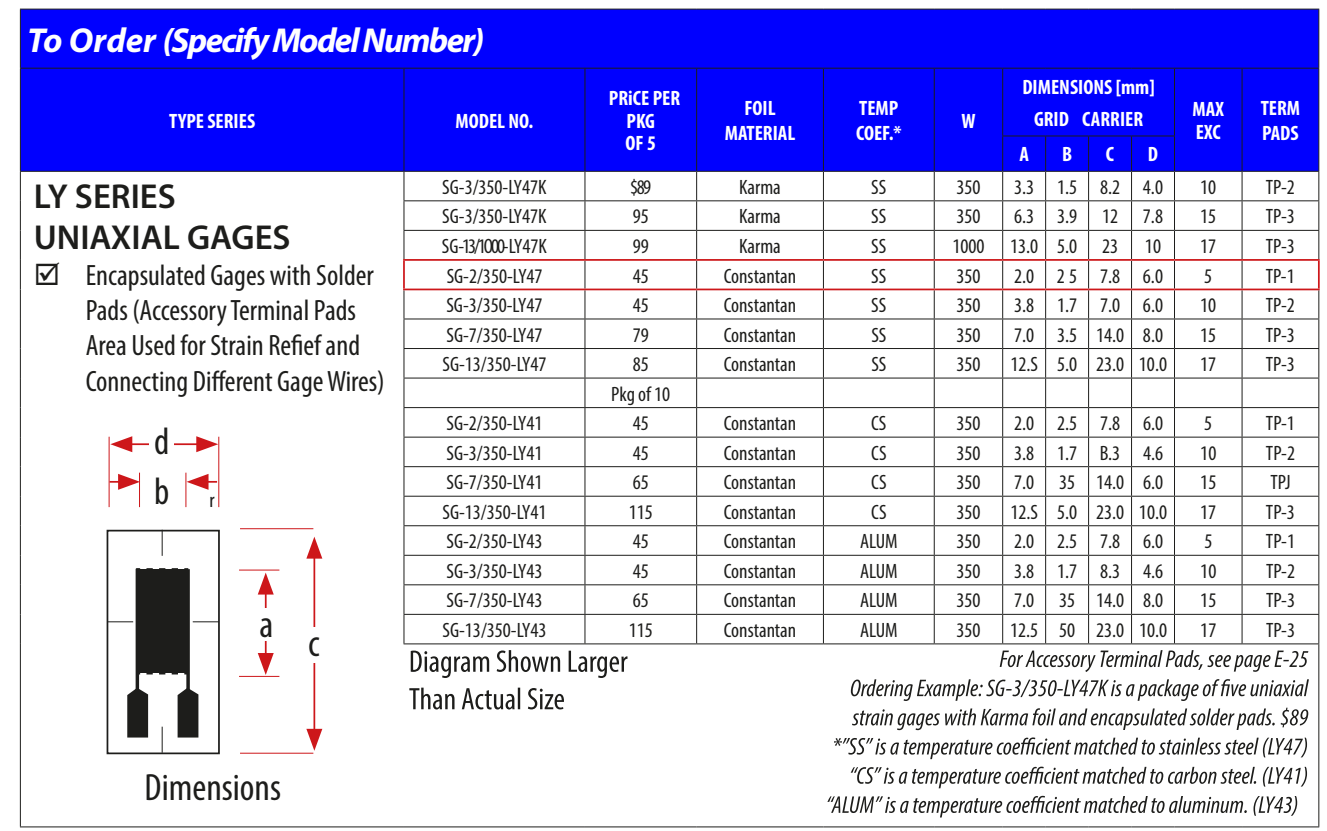

Figura 14. Galga extensiométrica recomendada.

Fuente: [18]

Para el cálculo de las fuerzas horizontales y verticales de operación debido a la interacción suelo-herramienta, se utilizan las ecuaciones 6 y 7.

$$
\begin{gathered}
H=P \sin (\alpha+\delta)+A_{d} d w \cot \alpha \\
V=P \cos (\alpha+\delta)-A_{d} d w
\end{gathered}
$$

Donde el coeficiente de adhesión $A_{d}$ y el ángulo de fricción $\delta$ suelo-herramienta son registrados por el operario; los datos geométricos que aparecen en las ecuaciones quedan definidos por los cuerpos del subsolador y la fuerza $\mathrm{P}$ se halla del equilibrio de las fuerzas que se encuentran representadas en la figura 15. 


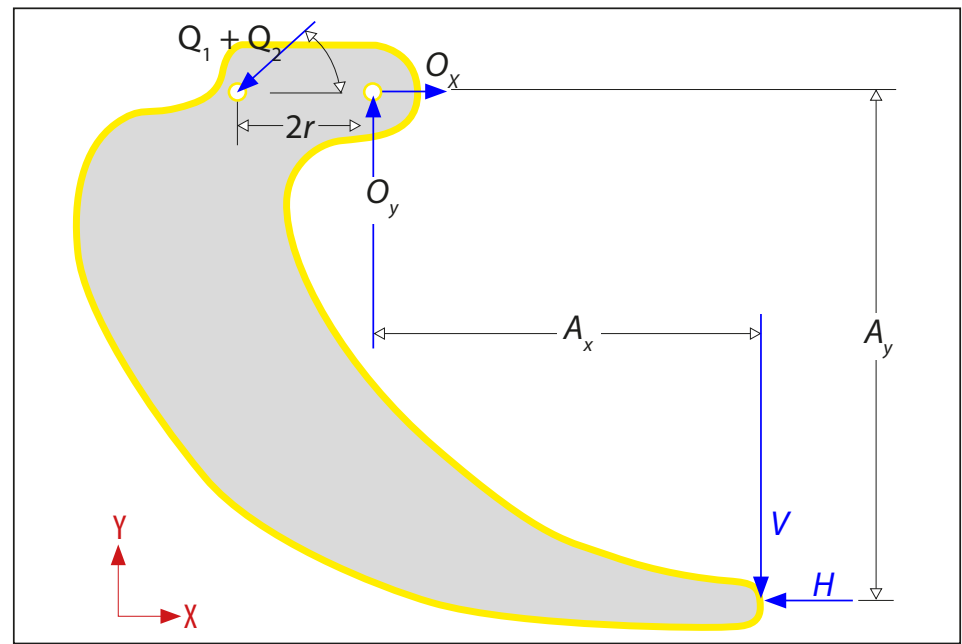

Figura 15. Fuerza H y V en función de Q.

Fuente: elaboración propia

Haciendo equilibrio de momentos en el punto $O$ se obtiene (8):

$$
\sum M_{O}=(2 r)\left(Q_{1}+Q_{2}\right) \sin (\gamma)-A_{x} V-A_{y} H=0
$$

Donde $\gamma$ es el peso específico del suelo, $r, A_{x}$ y $A_{y}$ son parámetros definidos por la geometría y estado de carga del cuerpo del subsolador (ver figura 16 y ecuaciones (9) y (10)).

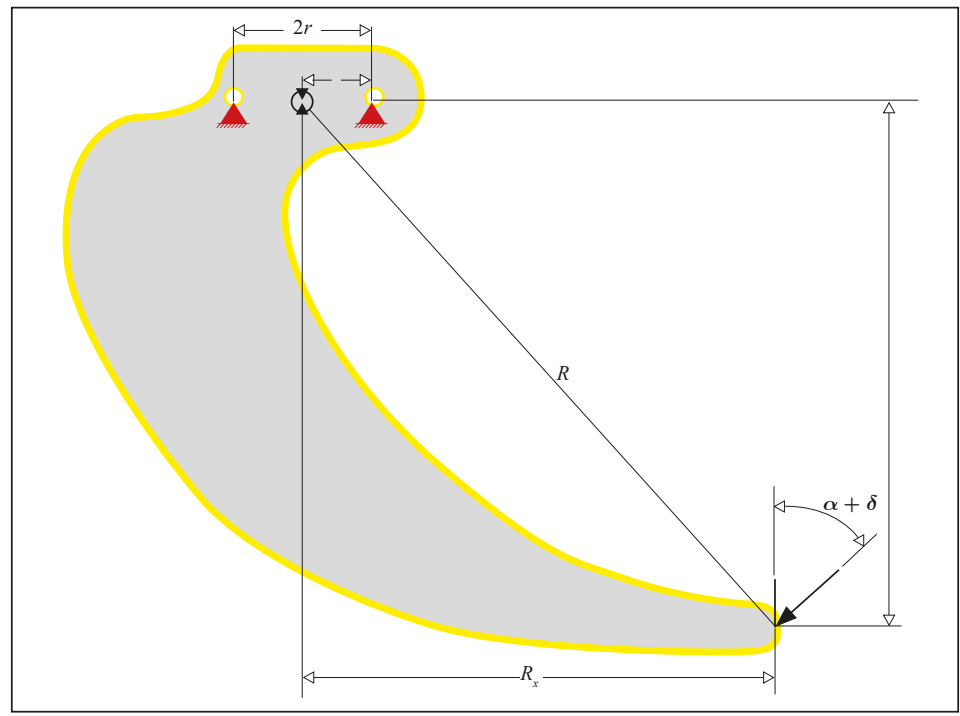

Figura 16. Diagrama de cuerpo libre del cuerpo del subsolador. 


$$
\begin{gathered}
A_{x}=R_{x}-r \\
A_{y}=R_{y}
\end{gathered}
$$

Considerando la fuerza de Recce y condiciones estáticas, se deriva $P$ en la ecuación (11).

$$
P=\frac{2 r\left(Q_{1}+Q_{2}\right) \sin (\gamma)+A_{d} d w\left[A_{x}-A_{y} \cot (\alpha)\right]}{A_{x} \cos (\alpha+\delta)+A_{y} \sin (\alpha+\delta)}
$$

Reemplazando la expresión de P de la ecuación (11) en las ecuaciones (6) y (7), se derivan las fuerzas que siente el subsolador debido al contacto con el suelo en función de las fuerzas medidas en las barras instrumentadas (ver ecuaciones (12) y (13)):

$$
\begin{gathered}
H=\frac{2 r\left(Q_{1}+Q_{2}\right) \sin (\gamma)+A_{d} d w\left[A_{x}-A_{y} \cot (\alpha)\right]}{A_{x} \cos (\alpha+\delta)+A_{y} \sin (\alpha+\delta)} \sin (\alpha+\delta)+A_{d} d w \cot \alpha(12) \\
V=\frac{2 r\left(Q_{1}+Q_{2}\right) \sin (\gamma)+A_{d} d w\left[A_{x}-A_{y} \cot (\alpha)\right]}{A_{x} \cos (\alpha+\delta)+A_{y} \sin (\alpha+\delta)} \cos (\alpha+\delta)-A_{d} d w
\end{gathered}
$$

Estas fuerzas son calculadas por el módulo controlador a fin de registrarlas en una memoria SD, si el operario da la entrada de control "inicio 2".

\section{CONCLUSIONES}

Este artículo presentó un procedimiento estructurado que permitió el diseño de un sistema instrumentado de seguridad de forma que se garantiza la operación continua de subsolado, independiente de la presencia de obstáculos. Además, el sistema ofrece la posibilidad de registro de las cargas de operación que permiten seleccionar otros dispositivos para la preparación del suelo.

El procedimiento permitió identificar que el equipo seleccionado contaba con un fusible mecánico no apto para proteger el subsolador.

Utilizando la alternativa de perno fusible para una condición de suelo seco, se determinaron los valores de esfuerzos críticos ante una sobrecarga en el cuerpo lateral, 215.32 $\mathrm{MPa}$, que fueron alcanzados para un ángulo de fricción de $11^{\circ} \mathrm{y}$ una fuerza reacción suelo-herramienta $\mathrm{P}$ de $25621 \mathrm{~N}$. 
El procedimiento integró herramientas de modelado como diagramas de casos de uso y de actividades UML y diagramas funcionales de IDEF0 para la definición de los requisitos funcionales del sistema.

Este trabajo evidenció la importancia de adaptaciones tecnológicas incorporando desarrollos de diferentes áreas, tales como agronomía, mecánica, electrónica, instrumentación y ciencias de la computación, a fin de hacer competitivo el rendimiento de la producción del campo.

\section{AGRADECIMIENTOS}

Se agradece a la Universidad del Valle por el soporte y al Ingenio de la región del Valle por el apoyo a esta investigación.

\section{REFERENCIAS}

[1] D. Grigg, "The transformation of agriculture in the West," Journal of Rural Studies, vol. 9, N. ${ }^{\circ}$, p. 199, April 1993.

[2] I. M. Johnston, "Classic Tractor Tales. The first tractor engines," The Australian Cottongrower, pp. 68-71, December 2003.

[3] J. Mejías, J. A. Martínez y I. Macías, "Impacto Ambiental De La Introducción De Un Prototipo Agrícola Para La Labranza De Conservación,” Revista DELOS: Desarrollo Local Sostenible, vol. 6, n. ${ }^{\circ}$ 16, pp. 1-14, February 2013.

[4] V. Ortiz, "Diseño, construcción y evaluación de un sistema de arado de discos reversible," Latacunga, Ecuador, 2008.

[5] A. Celik y R. L. Raper, "Design and evaluation of ground driven rotary subsoilers," Soil \& Tillage Research, vol. 124, pp. 203-210, August 2012.

[6] Y. You, D. Wang y J. Liu, "A divice for mechanical remediation of degraded grasslands," Soil \& Tillage Research, vol. 118, pp. 1-10, January 2012.

[7] J. P. Corredor, "Desarrollo de un sistema de control en la aplicación de técnicas selectivas de eliminación de maleza,” Bogotá, 2011.

[8] M. F. Polanco, Maquinaria y mecanización agrícola. Universidad Abierta y a Distancia 2007.

[9] J. Stafford, "The performance of a rigid tine in relation to soil properties and speed," Journal of Agricultural Engineering Research, vol. 24, n. ${ }^{\circ}$ 1, pp. 41-56, March 1979.

[10] J. Stafford, "Force prediction models for brittle and flow failure of soil by draught tillage tools," Journal of Agricultural Engineering Research, vol. 29, n. . 1, pp. 51 -60, January 1984.

[11] D. Hettiaratchi, B. Witney y A. Reece, "The calculation of passive pressure in twodimensional soil failure," Journal of Agricultural Engineering Research, vol. 11, nº 2, pp. 89- 107, June 1966. 
[12] D. Hettiaratchi y A. Reece, "Symmetrical three-dimensional soil failure," Journal of Terramechanics, vol. 4, n. ${ }^{\circ} 3$, pp. 45-67, 1967.

[13] “IEEE Standard for Funtional Modeling Language - Syntax and Semantic for IDEF0," IEEE Std 1320.1-1998, p. I, 1998.

[14] E. Hull, K. Jackson y J. Dick, Requirements Engineering, London: Springer, 2011.

[15] B. C. Falgueras, Ingeniería de software, UOC, 2002.

[16] C. Cassiolato, "smar," 3 July 2013. [En línea]. Available: http://www.smar.com/en/technicalarticles/article.asp?id=132. [Último acceso: 1 July 2014].

[17] ATOS, “ATOS on line,” [En línea]. Available: http://www.atos.com/espanol/technical_tables/ espanol/TF060.pdf. [Último acceso: 15 July 2014].

[18] OMEGA, “OMEGA," [En línea]. Available: http://www.omega.com/Pressure/pdf/XDUCER_GAGES.pdf. [Último acceso: 20 July 2014]. 